\title{
Three-Dimensional Measures of Bony Resection During Femoral Osteochondroplasty Are Related to Alpha Angle Measures: A Cadaveric Study
}

\author{
Thomas D. Alter, M.S., Philip Malloy, Ph.D., P.T., Alex C. Newhouse, B.S., \\ Sunikom Suppauksorn, M.D., Alejandro Espinzoa Orias, Ph.D., Jorge Chahla, M.D., Ph.D., \\ Nozomu Inoue, M.D., Ph.D., and Shane J. Nho, M.D., M.S.
}

\begin{abstract}
Purpose: To determine whether 3-dimensional (3D)-reconstructed proximal femoral bone models can be used to quantify femoral osteochondroplasty and to determine whether the 3D-based metrics are related to clinical alpha angle measures. Methods: Six cadaveric specimens with cam-type morphology underwent open femoral osteochondroplasty. Alpha angles were measured on the oblique axial computed tomography slice before and after femoral osteochondroplasty. Preoperative and postoperative computed tomography-based 3D reconstructed femur models were generated for each cadaveric specimen. A 3D-3D registration technique was used to merge the preoperative and postoperative models to measure the surface-to-surface distance between the model surfaces. Bivariate correlation analyses were used to determine the correlations between the preoperative, and the difference between the preoperative and postoperative alpha angle ( $\Delta$ alpha angle) measures and each of the femoral osteochondroplasty variables of surface area $\left(\mathrm{mm}^{2}\right)$, volume $\left(\mathrm{mm}^{3}\right)$, maximum height $(\mathrm{mm})$, and mean height $(\mathrm{mm})$. The strength of the bivariate correlations was defined as follows: weak 0.1 to 0.3, moderate 0.3 to 0.5, and strong as 0.5 to 1.00. Results: Bivariate correlations revealed a strong positive correlation between preoperative alpha angle with femoral osteochondroplasty volume $(\mathrm{r}=0.899, P=.007)$ and surface area $(\mathrm{r}=0.899, P=.007)$. No significant correlations were found between the change in alpha angle and the osteochondroplasty variables. Conclusions: In this study, pre- and postoperative 3D bone models could be used to quantify femoral osteochondroplasty and to determine if the 3D-based metrics are related to clinical alpha angle measures. Clinical Relevance: 3D-reconstructed image bone models may be helpful to ensure that adequate femoroplasty is performed intraoperatively, in particular with arthroscopic approach in which visualization may be challenging due to capsular management issues and surgeon experience.
\end{abstract}

$\mathbf{F}$ emoral osteochondroplasty for cam-type morphology is commonly performed during hip arthroscopy in patients with femoroacetabular impingement syndrome (FAIS) and has been shown to

From the Section of Young Adult Hip Surgery, Division of Sports Medicine, Department of Orthopedic Surgery, Hip Preservation Center, Rush University, Chicago, Illinois (T.D.A., P.M., A.C.N., S.S., A.E.O., J.C., N.I., S.J.N.); and Department of Physical Therapy, Arcadia University, Pennsylvania (P.M.), U.S.A.

The authors report the following potential conflicts of interest or sources of funding: A.E.O. reports nonfinancial support from PLoS One and other from Stryker, outside the submitted work. J.C. reports other from the American Orthopaedic Society for Sports Medicine, Arthrex, Arthroscopy Association of North America, CONMED Linvatec, International Society of Arthroscopy, Knee Surgery, and Orthopaedic Sports Medicine, Ossur, and Smith $\theta$ Nephew, outside the submitted work. N.I. reports other from the National Institutes of Health (NCCIH), outside the submitted work. S.J.N. receives research support from AlloSource; other from the American Orthopaedic Association and American Orthopaedic Society for Sports Medicine; nonfinancial support from Arthrex; other from the Arthroscopy Association of be an effective treatment to eliminate hip pain and improve functional status. ${ }^{1-6}$ It is of prime importance for surgeons to perform a comprehensive bony resection of cam-type morphology to achieve successful

North America; nonfinancial support from Athletico, DJ Orthopaedics, Linvatec, and Miomed; personal fees from Ossur; nonfinancial support from Smith $\theta$ Nephew; and personal fees from Springer and Stryker, outside the submitted work. No potential conflict reported by S.J.N. provided any form of funding or material or financial support for this work. Full ICMJE author disclosure forms are available for this article online, as supplementary material.

Received March 22, 2021; accepted August 19, 2021.

Address correspondence to Thomas Alter, 1611 W Harrison St, Chicago, IL 60612.E-mail: thomas.alter137@gmail.com

(C) 2021 THE AUTHORS. Published by Elsevier Inc. on behalf of the Arthroscopy Association of North America. This is an open access article under the CC BY-NC-ND license (http://creativecommons.org/licenses/by-nc-nd/4.0/). 2666-061X/21397

https://doi.org/10.1016/j.asmr.2021.08.016 
postoperative outcomes in these patients. Numerous studies have shown that residual cam-type morphology is the primary reason for revision hip arthroscopy. ${ }^{7-10}$ Typically, surgeons performing arthroscopic osteochondroplasty rely on a combination of intraoperative fluoroscopy and dynamic examination of the hip under direct visualization to assess adequate femoral resection. A current question that faces the field of hip arthroscopy is, "what defines an adequate femoral osteochondroplasty resection?"11 Unfortunately, the vast majority of studies that describe postoperative clinical outcomes fail to report the postoperative radiographic parameters, thereby adding to the challenge of establishing a metric for adequate bony resection in patients with FAIS with cam-type morphology.

The alpha angle (AA) is the primary metric for defining the severity of cam-type morphology and is used to evaluate bony resection following hip arthroscopy. AA measures have been established using a variety of imaging modalities and can be assessed on multiple imaging views. ${ }^{12-19}$ However, a threshold to define pathologic morphology on each imaging modality, as well as the most appropriate view for a comprehensive cam assessment, is still under debate. Although Nötzli et al. ${ }^{20}$ have suggested that a postoperative AA cutoff of $42^{\circ}$ should be used to define adequate bony resection of cam-type morphology, a universally agreed-on and standardized threshold is also lacking.

Although its clinical use is primarily qualitative for preoperative planning, 3-dimensional (3D) image reconstruction methods for the quantification of femoral morphology also have been established. ${ }^{21,22}$ The 3D volumetric quantification of cam-type morphology has been shown to demonstrate better inter- and-intraobserver reliability than the AA measure, which reveals the potential importance of these measures for clinical practice. The primary application of 3D-reconstructed images has been for surgical planning; however, these techniques also may provide avenues for the development of methods to improve the quantification of osseous pathomorphology, such as cam-type FAIS. Therefore, 3D-reconstructed image models may offer valuable insight regarding important aspects of these pathologic 3D morphologies that are not yet well recognized with existing imaging measures. . $^{18,23,24}$

Image registration techniques are useful for comparing 3D models generated from different imaging modalities, such as computed tomography (CT) or magnetic resonance imaging (MRI) scans. The method Ochia et al., ${ }^{25}$ named volume merge, has been validated for use in a number of musculoskeletal applications. These $3 \mathrm{D}-3 \mathrm{D}$ registration techniques also allow for the ability to compare the differences between 2 merged 3D surface models, thereby being able to compare differences in surface shape between the models. Malloy et al. ${ }^{18}$ used these techniques to validate the use of 1.5T MRI scans to generate accurate 3D models, which provides the opportunity for the assessment of femoral morphology without the need to expose patients to ionizing radiation. These findings have both profound preoperative and postoperative clinical implications, and, combined with 3D modeling techniques, open the door to provide a deeper understanding of these abnormal 3D morphologies and the relationship between femoral structure and clinical imaging measures. The purposes of this study were to determine whether 3D-reconstructed proximal femoral bone models could be used to quantify femoral osteochondroplasty and whether the 3D-based metrics are related to clinical AA measures. We hypothesized that a larger preoperative alpha angle would be correlated with a greater amount of bone surface area and volume resected during an open femoral osteochondroplasty.

\section{Methods}

\section{Cadaveric Specimens}

The present study was entirely cadaveric. As such, institutional review board approval was not a study requirement. Six fresh-frozen cadaveric hemipelvises with radiographic evidence of cam-type morphology defined by an $\mathrm{AA}>55^{\circ}$ and no evidence of hip osteoarthritis defined by a Tönnis grade $>1$ were procured through an accredited tissue bank (ScienceCare, Phoenix, AZ). The exclusion criteria were history of metastatic cancer to the bones and previous surgical intervention performed on the pelvis or proximal femur. The sample size was based on a previous similar investigation. ${ }^{18}$ All specimens were evaluated by 2 orthopaedic surgeons (S.N. and S.S.), and no specimens were excluded from the study. The donor age and body mass index averages were $62.6 \pm 18.0$ years and $25.4 \pm$ $7.8 \mathrm{~kg} / \mathrm{m}^{2}$, respectively. All cadaveric specimens were stored in a laboratory freezer at $-20^{\circ} \mathrm{C}$ and were removed 48 hours before imaging and testing to ensure complete thawing.

\section{CT Image Acquisition and Segmentation}

Each intact thawed cadaveric hemipelvis underwent preoperative and postoperative CT imaging (GE Healthcare; BrightSpeed, Waukesha, WI). The CT scan acquisition used the following scan parameters: $120 \mathrm{kV}$, $250 \mathrm{mAs}$, slice thickness of $0.625 \mathrm{~mm}$, and $512 \times 512$ acquisition matrix. All CT images were exported as Digital Imaging and Communication in Medicine files and stored in our institutional Pictures Archiving and Communication System. A semi-autosegmentation process was performed using commercially available segmentation software (Mimics, version 21; Materialise 
Research, Leuven, Belgium). All segmentations were performed by a trained biomedical engineer. CT-based 3D-reconstructed hip models were produced and reviewed to plan the femoral osteochondroplasty.

\section{Open Hip Femoral Osteochondroplasty}

Following preoperative CT imaging, tissue removal was performed through dissection beginning in the prone position. All extracapsular soft tissues were dissected from each specimen, the joint capsule was removed, and the labrum was left intact. The cam morphology was assessed on preoperative scans and the deformity was outlined using a surgical marking pen before surgery and reviewed by 2 orthopaedic surgeons (SS and SJN). A single orthopaedic surgeon (SS) performed all operative procedures. A 5.5-mm burr was used to resect the identified cam lesion. After each specimen underwent open femoral osteochondroplasty, a postoperative CT scan was performed on the femoral specimen. The postoperative 3D reconstructed femoral models were produced and reviewed to ensure complete resection of abnormal bony morphology.

\section{Femoral AA Measures}

The preoperative and postoperative AA were measured on oblique axial CT slices as previously described. ${ }^{20}$ To summarize, the oblique axial sequence slice was chosen when the center of the femoral neck was best visualized while maintaining circumferential roundness of the femoral head. A Mose circle was drawn around the sclerotic region of the femoral head. A line was then drawn from the center of the Mose circle through the middle of the femoral neck. A second line was then drawn from the center of the Mose circle to the point where the femoral head loses sphericity with respect to the Mose circle. The angle between both lines forms the AA (Fig 1). The change in AA following the open femoral osteochondroplasty was calculated as the difference between preoperative AA and postoperative AA.

\section{Femoral Osteochondroplasty Measures Based on 3D-Reconstructed CT Femoral Surface Models}

3D geometry of the pre- and postoperative femoral osteochondroplasty CT models was compared by measuring surface-to-surface least distance distribution between each pair of superimposed models. The surface-to-surface subtraction algorithm was previously used to validate preoperative 1.5T MRI proximal femoral bone models, which demonstrated absolute agreement with bone models generated by CT imaging. ${ }^{18}$ In this study, the same surface to surface comparison was used, with the addition of an intervention to compare the preoperative and postoperative surfaces and quantify the boney resection. Superimposition of the two 3D models was performed by 3D-3D

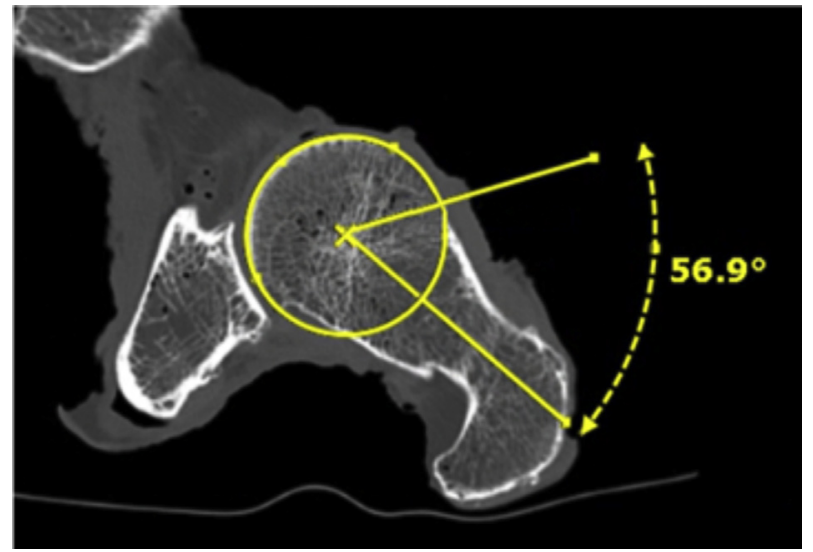

Fig 1. Alpha angle measurement on the oblique axial slice of computed tomography scan.

registration using a validated volume merge method (accuracy, translation: $0.1 \mathrm{~mm}$, rotation: $\left.0.2^{\circ}\right) .{ }^{25}$ The surface-to-surface least distance between the two 3D models was calculated by a point-to-surface distance calculation algorithm using a custom-written program coded in Microsoft Visual C++ 2015 with Microsoft Foundation Class programming environment (Microsoft Corp., Redmond, WA). The femoral osteochondroplasty region was defined as a $3 \mathrm{D}$ space at the head-neck junction created by surfaces of pre- and postfemoral osteochondroplasty models with a gap distance over $0.5 \mathrm{~mm}$ considering the CT resolution. The femoral osteochondroplasty surface area was defined as the surface area of the postosteochondroplasty surface (Fig 2).

\section{Statistical Analysis}

Before analysis, all data were inspected to evaluate the assumptions for parametric statistical analysis. In cases of assumption violation, the nonparametric statistical analysis tests were performed. The variables of interest in the current study were the AA measured before (pre-AA) and after (post-AA) the osteochondroplasty and the change ( $\triangle \mathrm{AA})$ in the alpha angle, and the femoral osteochondroplasty measures of: femoral osteochondroplasty volume $\left(\mathrm{mm}^{3}\right)$, osteochondroplasty surface area $\left(\mathrm{mm}^{2}\right)$, maximum osteochondroplasty height (FH max; $\mathrm{mm}$ ), and mean osteochondroplasty height (FH mean; mm). A paired samples $t$ test was used to evaluate mean differences in AAs before and after open femoral osteochondroplasty. Pearson's $(r)$ product moment coefficients and Spearman rank coefficients were used to determine the correlations between the preoperative, and the difference between the preoperative and postoperative alpha angle ( $\Delta$ AA) measures and each of the femoral osteochondroplasty variables. All data are reported as means and standard deviations. A significance level of 


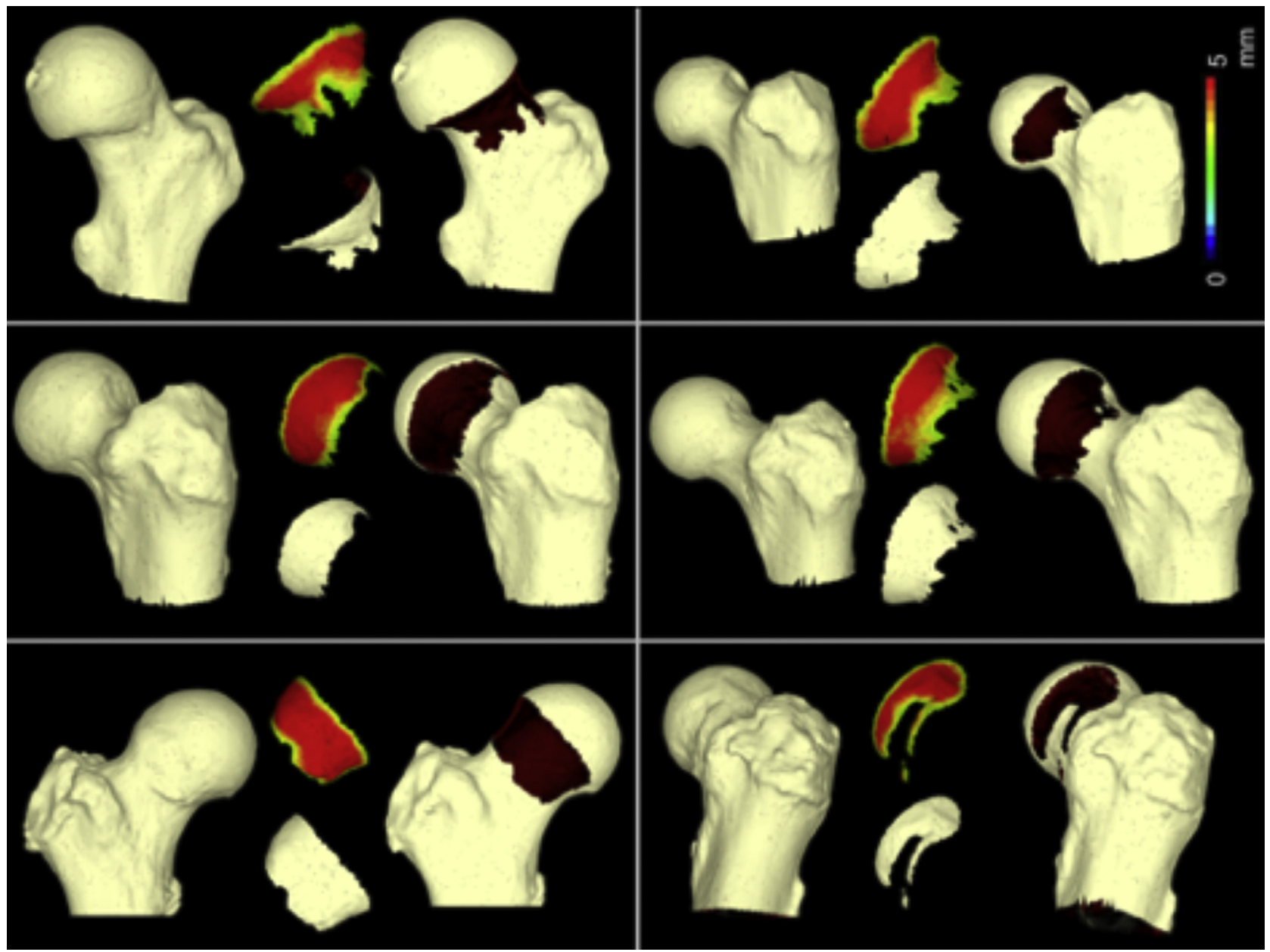

Fig 2. Preoperative and postoperative 3-dimensional-reconstructed hip models of cadaveric specimen with application of the image subtraction analysis quantifying femoral osteochondroplasty volume, femoral osteochondroplasty surface area, and femoral osteochondroplasty max and min height. A scale (top right corner) demonstrates the surface-to-surface difference measured in millimeters between preoperative and postoperative bone models with blue representing minimal resection and red indicating greater than $5 \mathrm{~mm}$ of resection.

$\alpha=0.05$ using a 2 -tailed analysis was used to determine statistical significance. The strength of the bivariate correlations were defined as follows: weak 0.1 to 0.3 , moderate $>0.3$ to $<0.5$, and strong as 0.5 to $1.00 .{ }^{26}$ All statistical analysis was performed using SPSS (version 26; IBM, Armonk, NY).

\section{Results}

There was a significant decrease in the AA after open femoral osteochondroplasty (preoperative $61.7 \pm 6.0^{\circ}$ vs postoperative $38.0 \pm 5.2^{\circ} ; P<.001$ ). The preoperative and postoperative AAs and open femoral osteochondroplasty measures are displayed in Table 1. There were significant positive correlations between preoperative AAs and the osteochondroplasty measures of volume and surface area resected. No significant correlations were found between the change in AA and the osteochondroplasty variables (Table 2 ).

\section{Discussion}

In this study, we found that the preoperative AA measured on an oblique axial-view CT scan demonstrated strong positive correlations with amount of bone surface area and volume resected during an open femoral osteochondroplasty. These observed in vitro findings reveal an important association between what the surgeon visually defines as cam-type pathomorphology, and a common clinical imaging measure that is used to identify cam morphology in a patient with FAIS. However, in vivo studies are needed to further investigate this link using in vivo and arthroscopic femoral osteochondroplasty for cam-type morphology.

3D image evaluation of the hip in patients with FAIS could help to more clearly define a consistent definition for cam-type pathomorphology. Although our findings provide important preliminary evidence of a link between clinical measure of the preoperative AA and changes in the actual bone surface during surgery, it 
Table 1. Variables of Interest

Preoperative alpha Angle,
Postoperative alpha angle,
$\Delta$ alpha angle,
FSA, ${ }^{\circ} m^{2}$
FV, $\mathrm{mm}^{3}$
FH mean, mm
FH max, mm

NOTE. Values are mean \pm standard deviation.

$\Delta$ alpha angle $=$ change in alpha angle from preoperative to postoperative;

FH max, femoral osteochondroplasty maximum height; FH mean, femoral osteochondroplasty mean height; FSA, femoral osteochondroplasty surface area; FV, femoral osteochondroplasty volume.
Table 2. Bivariate Correlations Between Preoperative, and Alpha Angle Delta and All Femoral Osteochondroplasty Variables.

\begin{tabular}{lccccc}
\hline & \multicolumn{2}{c}{ Preoperative Alpha Angle, } & & \multicolumn{2}{c}{$\Delta$ Alpha Angle, } \\
\cline { 2 - 3 } \cline { 5 - 6 } & $\mathrm{r}$ & $P$ Value & & $\mathrm{r}$ & $P$ Value \\
\hline FSA, mm & 0.899 & $.007^{*}$ & & 0.294 & .286 \\
$\mathrm{FV}, \mathrm{mm}^{3}$ & 0.899 & $.007^{*}$ & & 0.294 & .286 \\
$\mathrm{FH} \mathrm{max}, \mathrm{mm}$ & 0.600 & .104 & & 0.348 & .250 \\
$\mathrm{FH}$ mean, mm & 0.551 & .129 & & 0.294 & .286 \\
\hline$\Delta$ alpha angle & $=$ change in alpha & angle from & preoperative to \\
postoperative. & & & \\
FH max, femoral osteochondroplasty maximum height; FH mean, \\
femoral osteochondroplasty mean height; FSA, femoral osteochon- \\
droplasty surface area; FV, femoral osteochondroplasty volume. \\
*Indicates statistical significance at .05.
\end{tabular}

There is clear evidence that surgical intervention for FAIS demonstrates very good-to-excellent short- to mid-term outcomes in regards of pain reduction, return to function, and patient satisfaction..$^{3,4,27-31}$ Although clinical outcomes of long-term studies continue to show positive outcomes, the field of hip preservation must continue to strive for a better understanding of the role of the 3D morphology in the development of hip pain and symptoms to best select in patients with FAIS appropriate for surgery. Interestingly, a recent study found that a volumetric analysis of cam morphology demonstrated superior reliability to the AA measure and that these $3 \mathrm{D}$ measures were significantly correlated with the extent of labral tearing whereas the alpha angle was not related to the labral tear extent. ${ }^{19}$ This association between 3D morphology and associated joint injury highlights the need for a better understanding of the 3D nature of these morphologies as they relate to causing joint damage and the development of symptoms. The findings from the current study add to previous studies using 3D analysis methods and demonstrate that there is an association between the complex 3D aspects of cam morphology and the 2D measures we use to evaluate this 3D hip disorder in the clinical setting.

\section{Limitations}

As is the case with any study, a number of limitations must be considered. This was an exploratory study using 3D image-based analysis methods, therefore we used a small sample of 6 cadaveric hemipelvis specimens. These specimens also were used as part of a larger study investigating surgical cam resection. This small sample may elevate the risk of type II error. However, the sample size used in the current study was consistent with similar in vitro cadaveric studies. ${ }^{32-36}$ The 3D femoroplasty metrics required both preoperative and postoperative CT imaging. CT is not routinely performed postoperatively due to radiation exposure; however, previous studies have demonstrated absolute clinical reason for comprehensive evaluation also is
related to patient selection for surgical intervention. 
agreement between CT and MRI proximal femoral bone models, demonstrating long term potential clinical utility. ${ }^{18}$ Hip arthroscopy has become increasingly popular over open approaches for the treatment of FAIS. Although no longer the clinic norm, the present study was performed in the open setting. Future studies a warranted in the arthroscopic setting to demonstrate the in vivo application of this technique. However, this approach allowed for direct visualization of the bony deformity and both preoperative and postoperative proximal femoral bone models can be produced in the arthroscopic setting as well. The $2 \mathrm{D}$ alpha angle was only measured on the oblique axial slice of the CT. Future studies should include AA measures based on commonly ordered clinical screening radiographs or $3 \mathrm{D}$ bone model-based radial sequence measures. In addition, we did not account for femoral head size between specimens in the osteochondroplasty analysis results. Previous studies have found that femoral head volumes were significantly larger for male than female subjects and correlated with increasing patient height. ${ }^{37,38}$ Theoretically, a larger femoral head should require a greater amount of bony resection per degree change in AA. Future studies may, therefore, control for femoral head volume in reporting osteochondroplasty volume as a function of the size of the femoral head.

\section{Conclusions}

In this study, pre- and postoperative 3D bone models could be used to quantify femoral osteochondroplasty and to determine whether the 3D-based metrics are related to clinical AA measures.

\section{References}

1. Hoch A, Schenk P, Jentzsch T, Rahm S, Zingg PO. FAI morphology increases the risk for osteoarthritis in young people with a minimum follow-up of 25 years. Arch Orthop Trauma Surg 2021;141:1175-1181.

2. Wyles CC, Norambuena GA, Howe BM, et al. Cam deformities and limited hip range of motion are associated with early osteoarthritic changes in adolescent athletes: A prospective matched cohort study. Am J Sports Med 2017;45:3036-3043.

3. Menge TJ, Briggs KK, Dornan GJ, McNamara SC, Philippon MJ. Survivorship and outcomes 10 years following hip arthroscopy for femoroacetabular impingement: Labral debridement compared with labral repair. J Bone Joint Surg Am 2017;99:997-1004.

4. Lansdown DA, Kunze K, Ukwuani G, Waterman BR, Nho SJ. The importance of comprehensive cam correction: Radiographic parameters are predictive of patientreported outcome measures at 2 years after hip arthroscopy. Am J Sports Med 2018;46:2072-2078.

5. Levy DM, Kuhns BD, Chahal J, Philippon MJ, Kelly BT, Nho SJ. Hip arthroscopy outcomes with respect to patient acceptable symptomatic state and minimal clinically important difference. Arthroscopy 2016;32:1877-1886.
6. Nasser R, Domb B. Hip arthroscopy for femoroacetabular impingement. EFORT Open Rev 2018;3:121-129.

7. Shapira J, Kyin C, Go C, et al. Indications and outcomes of secondary hip procedures after failed hip arthroscopy: A systematic review. Arthroscopy 2020;36: 1992-2007.

8. Sardana V, Philippon MJ, de Sa D, et al. Revision hip arthroscopy indications and outcomes: A systematic review. Arthroscopy 2015;31:2047-2055.

9. O'Connor M, Steinl GK, Padaki AS, Duchman KR, Westermann RW, Lynch TS. Outcomes of revision hip arthroscopic surgery: A systematic review and metaanalysis. Am J Sports Med 2020;48:1254-1262.

10. Ross JR, Larson CM, Adeoye O, Kelly BT, Bedi A. Residual deformity is the most common reason for revision hip arthroscopy: A three-dimensional CT study. Clin Orthop Relat Res 2015;473:1388-1395.

11. van Klij P, Reiman MP, Waarsing JH, et al. Classifying cam morphology by the alpha angle: A systematic review on threshold values. Orthop J Sports Med 2020;8: 2325967120938312.

12. Konan S, Rayan F, Haddad FS. Is the frog lateral plain radiograph a reliable predictor of the alpha angle in femoroacetabular impingement? J Bone Joint Surg $\mathrm{Br}$ 2010;92:47-50.

13. Golfam M, Di Primio LA, Beaule PE, Hack K, Schweitzer ME. Alpha angle measurements in healthy adult volunteers vary depending on the MRI plane acquisition used. Am J Sports Med 2017;45:620-626.

14. Beaule PE, Zaragoza E, Motamedi K, Copelan N, Dorey FJ. Three-dimensional computed tomography of the hip in the assessment of femoroacetabular impingement. J Orthop Res 2005;23:1286-1292.

15. Kobayashi N, Sumi K, Higashihira S, et al. Correlations and reproducibility between radiographic and radial alpha angles in the evaluation of cam morphology. Orthop $J$ Sports Med 2020;8:2325967120932922.

16. Samim M, Eftekhary N, Vigdorchik JM, et al. 3D-MRI versus $3 \mathrm{D}-\mathrm{CT}$ in the evaluation of osseous anatomy in femoroacetabular impingement using Dixon 3D FLASH sequence. Skeletal Radiol 2019;48:429-436.

17. Audenaert EA, Baelde N, Huysse W, Vigneron L, Pattyn C. Development of a three-dimensional detection method of cam deformities in femoroacetabular impingement. Skeletal Radiol 2011;40:921-927.

18. Malloy P, Gasienica J, Dawe R, et al. 1.5 T magnetic resonance imaging generates accurate $3 \mathrm{D}$ proximal femoral models: Surgical planning implications for femoroacetabular impingement. J Orthop Res 2020;38: 2050-2056.

19. Dessouky R, Chhabra A, Zhang L, et al. Cam-type femoroacetabular impingement-correlations between alpha angle versus volumetric measurements and surgical findings. Eur Radiol 2019;29:3431-3440.

20. Nötzli HP, Wyss TF, Stoecklin CH, Schmid MR, Treiber K, Hodler J. The contour of the femoral head-neck junction as a predictor for the risk of anterior impingement. $J$ Bone Joint Surg Br 2002;84:556-560.

21. Atkins PR, Shin Y, Agrawal P, et al. Which twodimensional radiographic measurements of cam femoroacetabular impingement best describe the three- 
dimensional shape of the proximal femur? Clin Orthop Relat Res 2019;477:242-253.

22. Harris MD, Datar M, Whitaker RT, Jurrus ER, Peters CL, Anderson AE. Statistical shape modeling of cam femoroacetabular impingement. J Orthop Res 2013;31: $1620-1626$.

23. Larson CM. Editorial commentary: "The earth is not flat": Progressing from plain radiographs to three-dimensional imaging when evaluating hip disorders. Arthroscopy 2020;36:2633-2634.

24. Irie $\mathrm{T}$, Espinoza Orias AA, Irie TY, et al. Computed tomography-based three-dimensional analyses show similarities in anterosuperior acetabular coverage between acetabular dysplasia and borderline dysplasia. Arthroscopy 2020;36:2623-2632.

25. Ochia RS, Inoue N, Renner SM, et al. Three-dimensional in vivo measurement of lumbar spine segmental motion. Spine (Phila Pa 1976) 2006;31:2073-2078.

26. Cohen J. The effect size index: D. Statistical power analysis for the behavioral sciences, 2nd ed. Hillsdale, NJ: Lawrence Erlbaum Associates, 1988.

27. Cvetanovich GL, Weber AE, Kuhns BD, et al. Hip arthroscopic surgery for femoroacetabular impingement with capsular management: Factors associated with achieving clinically significant outcomes. Am J Sports Med 2018;46:288-296.

28. Kuhns BD, Hannon CP, Makhni EC, et al. A Comparison of clinical outcomes after unilateral or bilateral hip arthroscopic surgery: Age- and sex-matched cohort study. Am J Sports Med 2017;45:3044-3051.

29. Levy DM, Kuhns BD, Frank RM, et al. High rate of return to running for athletes after hip arthroscopy for the treatment of femoroacetabular impingement and capsular plication. Am J Sports Med 2017;45: $127-134$.

30. Kunze KN, Leong NL, Beck EC, Bush-Joseph CA, Nho SJ. Hip arthroscopy for femoroacetabular impingement improves sleep quality postoperatively. Arthroscopy 2019:35:461-469.

31. Griffin DR, Dickenson EJ, Wall PDH, et al. Hip arthroscopy versus best conservative care for the treatment of femoroacetabular impingement syndrome (UK FASHIoN): A multicentre randomised controlled trial. Lancet 2018;391:2225-2235.

32. Yanke AB, Shin JJ, Pearson I, et al. Three-dimensional magnetic resonance imaging quantification of glenoid bone loss is equivalent to 3-dimensional computed tomography quantification: Cadaveric study. Arthroscopy 2017;33:709-715.

33. Neubert A, Wilson KJ, Engstrom C, et al. Comparison of 3D bone models of the knee joint derived from CT and 3T MR imaging. Eur J Radiol 2017;93:178-184.

34. Lockard CA, Stake IK, Brady AW, et al. Accuracy of MRIbased talar cartilage thickness measurement and talus bone and cartilage modeling: Comparison with groundtruth laser scan measurements. Cartilage 2020: 1947603520976774.

35. Suppauksorn S, Beck EC, Rasio J, et al. A cadaveric study of cam-type femoroacetabular impingement: Biomechanical comparison of contact pressures between cam morphology, partial femoral osteoplasty, and complete femoral osteoplasty. Arthroscopy 2020;36:2425-2432.

36. Logishetty K, van Arkel RJ, Ng KCG, MuirheadAllwood SK, Cobb JP, Jeffers JRT. Hip capsule biomechanics after arthroplasty: The effect of implant, approach, and surgical repair. Bone Joint J 2019;101-B:426-434.

37. Zhang L, Wells JE, Dessouky R, et al. 3D CT segmentation of CAM type femoroacetabular impingement-reliability and relationship of CAM lesion with anthropomorphic features. Br J Radiol 2018;91:20180371.

38. Yanke AB, Khair MM, Stanley R, et al. Sex differences in patients with cam deformities with femoroacetabular impingement: 3-dimensional computed tomographic quantification. Arthroscopy 2015;31:2301-2306. 